\title{
RANDOM COMPLEX FEWNOMIALS, I
}

\author{
BERNARD SHIFFMAN AND STEVE ZELDITCH
}

\begin{abstract}
We introduce several notions of 'random fewnomials', i.e. random polynomials with a fixed number $f$ of monomials of degree $N$. The $f$ exponents are chosen at random and then the coefficients are chosen to be Gaussian random, mainly from the $\mathrm{SU}(m+1)$ ensemble. The results give limiting formulas as $N \rightarrow \infty$ for the expected distribution of complex zeros of a system of $k$ random fewnomials in $m$ variables $(k \leq m)$. When $k=m$, for $\mathrm{SU}(m+1)$ polynomials, the limit is the Monge-Ampère measure of a toric Kähler potential on $\mathbb{C P}^{m}$ obtained by averaging a 'discrete Legendre transform' of the Fubini-Study symplectic potential at $f$ points of the unit simplex $\Sigma \subset \mathbb{R}^{m}$.
\end{abstract}

\section{Contents}

Introduction 2

0.1. Fewnomial ensembles 3

0.2. Expected distribution of zeros $\quad 6$

0.3. Statement of results 6

0.4. More general toric weights 9

1. Preliminaries 10

1.1. The $\mathrm{SU}(m+1)$-ensembles 11

2. Fewnomial Ensembles 12

2.1. Precise definitions of random fewnomials 12

2.2. Expected zeros of fewnomial ensembles 13

2.3. Mass asymptotics and fewnomial Szegö kernels 14

2.4. Proof of Theorems 1 and $2 \quad 16$

3. Zeros of random fewnomial systems: Proof of Theorem 4 17

3.1. Computing the explicit formula: Proof of Corollary 5 19

4. General toric Kähler potentials 20

5. The $\mathbf{T}^{m}$ ensemble 21

References $\quad 22$

Date: May 30, 2022.

Research of the first author partially supported by NSF grant DMS-0901333; research of the second author partially supported by NSF grant DMS-0904252. 


\section{INTRODUCTION}

This article is concerned with the distribution of complex zeros of random systems of fewnomials. Fewnomials are polynomials

$$
\sum_{\alpha:|\alpha| \leq N} c_{\alpha} z^{\alpha}: \quad \#\left\{\alpha: c_{\alpha} \neq 0\right\}=f<<N,
$$

with a relatively small number $f<<N$ of non-zero coefficients in comparison to the degree. For instance, $z^{10,000}+5 z^{1,000}-3 z^{100}-1$ is a fewnomial but $z^{10,000}+z^{9,999}+\cdots+z+1$ is not. The fundamental idea is that the number of monomials, rather than the degree, measures the complexity of a polynomial system $[\mathrm{Kh}]$. The purpose of this article to begin an investigation of fewnomial complexity bounds from a probabilistic viewpoint. In this article, we introduce several natural ensembles of 'random fewnomial systems', and study the expected distribution of their complex zeros. In subsequent articles, we plan to study real zeros of real fewnomials and the more difficult problem of the correlations and variance of both real and complex zeros. The overall purpose is to study from a statistical point of view Khovanskii's bounds [Kh] on the Betti numbers of real algebraic varieties given by fewnomials and on the number of real zeros of a full fewnomial system. Statistical properties of the topology of real algebraic varieties given by non-fewnomial polynomials (i.e., with all the coefficients nonzero) have been studied, for example, in $[\mathrm{Bu}, \mathrm{Ro}, \mathrm{ShSm}, \mathrm{GW}]$ and the references in these papers, but not much is known statistically about zeros of fewnomials.

To put our problem into context, let us recall Khovanskii's theorem: Let $P=\left(P_{1}, \ldots, P_{m}\right)$ denote a system of $m$ complex polynomials on $\left(\mathbb{C}^{*}\right)^{m}$, and let $\Delta_{j}=\Delta_{P_{j}}$ denote the Newton polytope of $P_{j}$, i.e the convex hull of the exponents appearing non-trivially in $P_{j}$. Let $U \subset \mathbf{T}^{m}$ be an open set, where $\mathbf{T}^{m} \subset\left(\mathbb{C}^{*}\right)^{m}$ is the real $m$-torus, and let $N(P, U)$ be the number of zeros with arguments lying in $U$. When $U=\mathbf{T}^{m}, N(P, U)$ counts the total number of zeros in $\left(\mathbb{C}^{*}\right)^{m}$, which by the Bernstein-Kouchnirenko theorem [Be, Ko] can be expressed in terms of the mixed volume $V\left(\Delta_{1}, \ldots, \Delta_{m}\right)$. Given an angular sector determined by $U$, the number

$$
S(P, U):=V\left(\Delta_{1}, \ldots, \Delta_{m}\right) \operatorname{Vol}(U) / \operatorname{Vol}\left(\mathbf{T}^{m}\right)
$$

may be viewed as the 'average number' of complex zeros in the sector among random polynomial systems with the prescribed Newton polytopes $\Delta_{j}$. We denote this class of polynomial systems by

$$
P \in \operatorname{Poly}\left(\Delta_{1}, \ldots, \Delta_{m}\right):=\left\{\left(P_{1}, \ldots, P_{m}\right): \Delta_{P_{j}}=\Delta_{j}\right\} .
$$

Khovanskii's complex fewnomials theorem [Kh, $\S 3.13$, Th. 2] asserts that

$$
\sup _{P \in \operatorname{Poly}\left(\Delta_{1}, \ldots, \Delta_{m}\right)}|N(P, U)-S(P, U)| \leq \Pi\left(U, \Delta_{1}, \ldots, \Delta_{m}\right) \varphi(m, f)
$$

where $f$ is the number of non-zero coefficients of the system, and where $\Pi\left(U, \Delta_{1}, \ldots, \Delta_{m}\right)$ is the smallest number of translates of a certain region $\Delta^{*} \subset \mathbf{T}^{m}$ required to cover the boundary of $U$. One of the principal applications of this result is to give an upper bound for the number $N_{\mathbb{R}}(P)$ of real zeros of a fewnomial system: If $U_{j}$ is a sequence of small balls around $\{0\}$ shrinking to the point $\{0\}$, one has $\Pi=1$ and $S\left(P, U_{j}\right) \rightarrow 0$ and one obtains a bound of the form

$$
\left|N_{\mathbb{R}}(P)\right| \leq \varphi(m, f)
$$


entirely in terms of the number of non-zero monomials appearing in it and not its degree. We will refer to $f$ as the fewnomial number of the system.

Khovanskii's result may be interpreted in terms of the angular projection $\operatorname{Arg}:\left(z_{1}, \ldots, z_{m}\right)=$ $\left(\frac{z_{1}}{\left|z_{1}\right|}, \ldots, \frac{z_{m}}{\left|z_{m}\right|}\right)$ of the zero set to the real torus $\mathbf{T}^{m}$. His result (in the full system case) says that the angular projection of the fewnomial zero set is rather evenly distributed in $\mathbf{T}^{m}$. As a result, not too many zeros concentrate on the real set where $\theta=0$. Note that his measure of the concentration, taking the supremum in (2), is very astringent and is governed by the extreme cases. The idea of our work is to study its average value over fewnomial systems and polynomials.

The motivation for the statistical study is that the known estimates of $\varphi(m, f)$ are very large and are widely conjectured to overestimate the the bound by many orders of magnitude. Khovanskii's bound states that $\varphi(m, f) \leq 2^{m} 2^{f(f-1) / 2}(m+1)^{f}$. See [BBS, BRS] for relatively recent bounds and [Sot, St1, St2] for further background. A conjecture of Kouchnirenko, as corrected and refined by a number of people, states that the maximum number of real zeros in the positive real quadrant should be roughly $|f|^{2 m}$ where $|f|$ is the total number of monomials in the system. The uncertainty as to the true order of magnitude of $\varphi(m, f)$ suggests studying the bound probabilistically. The bound (3) resembles a variance estimate although it is measured in the much more difficult sup norm. It reflects the extremal behavior, which may only occur very rarely. This raises the question, what is the expected or average order of magnitude of the variance?

In this article we begin the study of random fewnomial systems by introducing several probability measures on spaces of complex fewnomials - i.e., on the set of pairs $(S, P)$ of spectra and polynomial systems with the given spectra. Our main results give the expected limit distribution of complex zeros in the ensembles. For example, Theorem 4 says that for a random system $\left(P_{1}^{N}, \ldots, P_{m}^{N}\right)$ of fewnomials on $\mathbb{C}^{m}$, each of fewnomial number $f$ and of degree $N$, where the exponents are chosen uniformly at random and the coefficients are chosen at random from the $\mathrm{SU}(m+1)$ ensemble (described below), the expected distribution of zeros in $\left(\mathbb{C}^{*}\right)^{m}$ is asymptotic to

$$
N^{m} \operatorname{det}_{1 \leq p, q \leq m}\left(\frac{\partial^{2}}{\partial \rho_{p} \partial \rho_{q}} \int_{\Sigma^{f}} \max _{j=1, \ldots, f}\left[\left\langle\rho, \lambda^{j}\right\rangle-\left\langle\widehat{\lambda^{j}}, \log \widehat{\lambda^{j}}\right\rangle\right] d \lambda^{1} \cdots d \lambda^{f}\right) d \rho_{1} \cdots d \rho_{m} \frac{d \theta_{1}}{2 \pi} \cdots \frac{d \theta_{m}}{2 \pi},
$$

where $z=\left(e^{\rho_{1} / 2+i \theta_{1}}, \ldots, e^{\rho_{m} / 2+i \theta_{m}}\right), \Sigma$ is the unit simplex in $\mathbb{R}^{m}$ with probability measure $d \lambda=m ! d \lambda_{1} \cdots d \lambda_{m}$, and $\widehat{\lambda}=\left(1-|\lambda|, \lambda_{1}, \ldots, \lambda_{m}\right)$.

0.1. Fewnomial ensembles. We consider several natural definitions which are motivated by different kinds of applications. More precise and detailed definitions are given in $\S 2$.

We denote the space of all complex holomorphic polynomials of degree $N$ by $\operatorname{Poly}(N)$. By the spectrum (or support) of a polynomial $P$, we mean the set $S_{P}$ of exponents of its non-zero monomials. We denote the space of polynomials with spectrum contained in $S$ by

$$
\operatorname{Poly}(S)=\left\{P\left(z_{1}, \ldots, z_{m}\right)=\sum_{\alpha \in S} c_{\alpha} \chi_{\alpha}(z), \quad \chi_{\alpha}(z):=z^{\alpha}\right\}, \quad S \subset \mathbb{N}^{m} .
$$

The Newton polytope of $P$ is the convex hull $\Delta_{P}$ of the spectrum $S_{P}$. More generally, we consider a system of $k \leq m$ polynomials $P_{1}, \ldots, P_{k}$ in $m$ complex variables, and write

$$
\operatorname{Poly}\left(S_{1}, \ldots, S_{k}\right)=\left\{\left(P_{1}, \ldots, P_{k}\right): P_{j} \in \operatorname{Poly}\left(S_{j}\right)\right\} \text {. }
$$


When $k=m$ we speak of a 'full' system, where the simultaneous zeros are almost always zero-dimensional.

In all of our definitions of random fewnomial system, the numbers $f_{j}$ of elements of each spectrum $S_{j}$ and the degrees $N$ of the polynomials $P_{j}$ are fixed. We then randomize with respect to the spectra $S_{j}$ and with respect to the coefficients $c_{j \alpha}$. With regard to the spectra, there are several natural choices of probability measure:

(I) Fixed spectrum up to dilation: Here, we fix a spectrum $S$, and then dilate it deterministically as the degree $N$ grows, i.e. scale $S \rightarrow N S$. This notion of random fewnomial is analogous to our notion of random polynomial with fixed Newton polytope in [SZ2]. The main difference is that the 'polytope' which we dilate is non-convex; indeed, it just consists of a fixed set of $f$ points. In fact, the techniques of [SZ2] generalize quite naturally to all non-convex polytopes. The only randomness is then with respect to the coefficients. The result is given in Theorem 1 .

(II) Dilates of a random spectrum from a polytope $\Delta$ : in this ensemble, we fix $\Delta$, choose the spectrum at random from $\Delta$ and then dilate the resulting spectrum. It is only a small step from case (I), but is apparently important in computational work. (We thank Maurice Rojas for emphasizing the interest of this case.) The result is given in Corollary 3.

(III) Random spectra of degree $N$ : At the opposite extreme, we may choose the spectra completely randomly (with respect to counting measure) from all possible $f$-element sets of exponents $\alpha \in \mathbb{N}^{m}$ of length $|\alpha|=\left|\alpha_{1}\right|+\cdots+\left|\alpha_{m}\right| \leq N$-i.e., subsets of the integral simplex $\mathbb{Z}^{m} \cap N \Sigma$, where $\Sigma=\left\{x \in \mathbb{R}^{m}: x_{j} \geq 0, \sum x_{j} \leq 1\right\}$ is the unit $m$-simplex, and $N \Sigma$ is its dilate by $N$. We put uniform measure on $\mathbb{Z}^{m} \cap N \Sigma$ and then choose spectra $S_{j} \subset \mathbb{Z}^{m} \cap N \Sigma$ of fixed cardinality $f$ independently and uniformly relative to counting measure. The result is given in Theorem 4 for $\mathrm{SU}(m+$ 1) fewnomials and in Theorem 7 for general toric Kähler potentials.

(IV) Random spectra contained in fixed Newton polytopes: Rather than just consider the simplex, and motivated by Khovanskii's variation formula, we now fix $k$ convex lattice polytopes $\Delta_{1}, \ldots, \Delta_{k}$ and then choose random spectra $S_{j} \subset \Delta_{j} \cap \mathbb{Z}^{m}$ with fixed cardinalities $f_{j}$ independently with uniform measures from these polytopes. We then replace the $\Delta_{j}$ by their dilations by $N \Delta_{j}$ to obtain higher degree ensembles. We note that the convex hull of $S_{j}$ is contained in $\Delta_{j}$ but equality rarely occurs. The result is given in Theorem 6 .

Having decided on an ensemble of spectra $\alpha$, we then define probability measures on the coefficients $c_{\alpha}$. We only consider Gaussian probability measures and make standard choices which are consistent with Khovanskii's bound. A key point is that Gaussian measures are determined by inner products in the space of polynomials. We choose the inner products as in $[\mathrm{SZ} 1, \mathrm{SZ} 3, \mathrm{SoZ2}, \mathrm{SoZ} 3]$ to be those $G_{N}(\varphi, \nu)$ of the weighted $L^{2}$ spaces $L^{2}\left(\mathbb{C P}^{m}, e^{-N \varphi} d \nu\right)$ of pluri-potential theory, which are specified by a Kähler potential $\varphi$ or Hermitian metric $h=e^{-\varphi}$ and a measure $d \nu$ on $\mathbb{C P}^{m}$. It is natural to restrict to $\varphi, \nu$ which are toric, i.e. invariant under the standard $\mathbf{T}^{m}$ torus action on $\mathbb{C P}^{m}$. Then the monomials $\left\{z^{\alpha}\right\}$ are always orthogonal and the Gaussian ensembles only differ in the $L^{2}$ - norms

$$
Q_{G_{N}(\varphi, \nu)}(\alpha)=\left\|z^{\alpha}\right\|_{G_{N}(\varphi, \nu)}^{2}=\int_{\mathbb{C}^{m}}\left|z^{\alpha}\right|^{2} e^{-N \varphi(z)} d \nu(z)
$$


of the monomials, viewed as homogeneous polynomials of degree $N$ (so that $|\alpha| \leq N$ ); equivalently, the Gaussian measures only differ in the variances of the coefficients in the monomial basis. We refer to $\S 1$ for details.

Since our emphasis is on the fewnomial aspects we only consider some basic examples of $(\varphi, \nu)$. In particular, we concentrate on the model case of $\mathrm{SU}(m+1)$ polynomials, where $\varphi(z)=\log \left(1+\|z\|^{2}\right)$ is the Fubini-Study potential and where $d \nu=\frac{1}{m !}\left(\frac{i}{2 \pi} \partial \bar{\partial} \varphi\right)^{m}$ is the Fubini-Study volume form.

Given the inner product $G_{N}(\varphi, \nu)$ underlying the Gaussian measure, we normalize the monomials to have $L^{2}$-norm equal to one, by putting

$$
\varphi_{\alpha}=\frac{z^{\alpha}}{Q_{G_{N}(\varphi, \nu)}(\alpha)},
$$

and then express polynomials of degree $N$ as the orthonormal sums

$$
P_{N}=\sum_{\alpha \in N \Sigma} c_{\alpha} \varphi_{\alpha}
$$

The Gaussian measure $\gamma_{N}$ induced by $G_{N}(\varphi, \nu)$ is defined by the condition that the $c_{\alpha}$ are independent complex normal variables of mean zero and variance one.

The Gaussian measure $d \gamma_{N}$ on $\operatorname{Poly}(N)$ induces conditional Gaussian measures $\gamma_{N \mid S}$ on the spaces $\operatorname{Poly}(S)$; i.e.,

$$
d \gamma_{N \mid S}\left(P_{N}\right)=\frac{1}{\pi^{|S|}} e^{-\sum\left|c_{\alpha}\right|^{2}} d c, \quad P_{N}=\sum_{\alpha \in S} c_{\alpha} \varphi_{\alpha}
$$

where $\varphi_{\alpha}$ is given by (7). Probabilities relative to $\gamma_{N \mid S}$ can be considered as conditional probabilities; i.e., for any event $E$,

$$
\operatorname{Prob}_{\gamma_{N}}\left\{P \in E \mid S_{P}=S\right\}=\operatorname{Prob}_{\gamma_{N \mid S}}(E) .
$$

We denote by $\mathbf{E}_{N \mid S}$ the expectation with respect to the conditional Gaussian measure $\gamma_{N \mid S}$. For a further discussion of conditional probabilities on polynomial (and more general) ensembles, see [SZZ]

Some of the possible (and well-studied) choices of the inner products and Gaussian measures are the following:

(a) The $\mathrm{SU}(m+1)$ ensembles defined above. On all of $\operatorname{Poly}(N)$ the expected distribution of zeros for each $N$ is uniform with respect to the $\mathrm{SU}(m+1)$-invariant volume form on $\mathbb{C P}^{m}$ (i.e. the Fubini-Study form).

(b) General toric Gaussian measures induced by $\mathbf{T}^{m}$-invariant Hermitian metrics $h=e^{-\varphi}$ on the line bundle $\mathcal{O}(1) \rightarrow \mathbb{C P}^{m}$ with positive curvature form $\omega_{\varphi}=i \partial \bar{\partial} \varphi$ (i.e. with a plurisubharmonic weight) and with $\nu=d V_{\varphi}:=\frac{1}{m !}\left(\frac{i}{2 \pi} \partial \bar{\partial} \varphi\right)^{m}$. We suppress geometric notions in this article, but state the general result in Theorem 7 (see $\S 4$ ).

(c) The $m$-dimensional Kac-Hammersley ensembles, where $Q_{N}(\alpha)=1$. Here, $\varphi \equiv 0$ (the opposite extreme from subharmonic weights) and $\nu=\delta_{\mathbf{T}^{m}}$. The norms of the monomials are independent of $N$ and only involve a fixed inner product on $\mathbb{C}^{m}$. In dimension one, the zeros of degree $N$ polynomials (with full spectrum $\mathbb{Z} \cap[0, N]$ ) concentrate on the unit circle as $N \rightarrow \infty$ [Ha], and in dimension $m$ the zeros of degree $N$ polynomials concentrate on the torus $\mathbf{T}^{m}$ [BS]. We briefly discuss this ensemble in $\S 5$. 
Remark: Khovanskii [Kh] and Kazarnovskii [Ka1, Ka2] consider ensembles where one fixes the spectra $S_{j}$ and chooses coefficients at random from the ensemble

$$
\mathbb{C} \mathbb{P}^{\left|S_{1}\right|-1} \times \mathbb{C P}^{\left|S_{2}\right|-1} \times \cdots \times \mathbb{C P}^{\left|S_{m}\right|-1},
$$

i.e., the product projective space of coefficients of polynomials with the prescribed spectra, equipped with the probability measure obtained by taking the product of (normalized) Fubini-Study volume measures on the factors. These ensembles amount to choosing the complex coefficients at random from the Euclidean spheres $S^{2\left|S_{j}\right|-1}$ and are easily seen to be equivalent to Gaussian random polynomials $\sum_{\alpha} c_{\alpha} z^{\alpha}$ with $c_{\alpha}$ independent complex normal variables of mean zero and variance one - i.e., they are equivalent to the Kac-Hammersley ensembles described above.

0.2. Expected distribution of zeros. Having fixed an ensemble of fewnomials, our interest is in the configuration of zeros

$$
Z_{P_{1}, \ldots, P_{k}}:=\left\{z \in\left(\mathbb{C}^{*}\right)^{m}: P_{1}(z)=\cdots=P_{k}(z)=0\right\}
$$

of a random fewnomial system with $k \leq m$. Here, $\mathbb{C}^{*}=\mathbb{C} \backslash\{0\}$. We refer to $k=1$ as the random fewnomial hypersurface case and to $k=m$ as the point case.

To each zero set we associate the current of integration $\left[Z_{P_{1}, \ldots, P_{k}}\right] \in \mathcal{D}^{\prime k, k}\left(\left(\mathbb{C}^{*}\right)^{m}\right)$ over the zeros of the system:

$$
\left(\left[Z_{P_{1}, \ldots, P_{k}}\right], \psi\right)=\int_{Z_{P_{1}, \ldots, P_{k}}} \psi, \quad \psi \in \mathcal{D}^{m-k, m-k}\left(\left(\mathbb{C}^{*}\right)^{m}\right) .
$$

In the point case, $\left[Z_{P_{1}, \ldots, P_{m}}\right]$ is obtained by putting point masses at each zero,

$$
\left[Z_{P_{1}, \ldots, P_{m}}\right]=\sum_{z \in Z_{P_{1}, \ldots, P_{m}}} \delta_{z},
$$

and the expected distribution is determined by the expected values of the random variables

$$
\mathcal{N}_{N}^{U}\left(P_{1}^{N}, \ldots, P_{m}^{N}\right):=\left[Z_{P_{1}, \ldots, P_{m}}\right](U)=\#\left\{z \in U: P_{1}^{N}(z)=\cdots=P_{m}^{N}(z)=0\right\}
$$

counting the number of zeros in an open set $U \subset\left(\mathbb{C}^{*}\right)^{m}$.

The expected distribution of zeros varies widely among the ensembles above. This is not surprising if one recalls, for instance, that zeros of random Kac polynomials concentrate on the unit circle, while those of $\mathrm{SU}(2)$ polyonomials are uniform with respect to the standard area form of $\mathbb{C P}^{1}=\mathbb{C} \cup \infty$, while those of polynomials with fixed Newton polytope have a forbidden region where zeros have an exotic concentration. In particular, the 'average number' $S(P, U)$ of zeros in the angular sector $U$ considered in Khovanskii's variance estimate (2) is itself a random variable which depends on the convex hull of the spectrum of $P$.

0.3. Statement of results. We will consider the zero distribution as a measure on $\left(\mathbb{C}^{*}\right)^{m}=$ $\mathbb{R}_{+}^{m} \times \mathbf{T}^{m}$ and denote points by $z=e^{\rho / 2+i \theta}$ in multi-index notation. Here, $\mathbf{T}^{m}$ denotes the real torus $\mathbf{T}^{m}=\left(S^{1}\right)^{m} \subset\left(\mathbb{C}^{*}\right)^{m}$. Given a locally bounded plurisubharmonic function $\varphi$ we denote by $\operatorname{MA}(\varphi)$ the associated Monge-Ampère measure

$$
\operatorname{MA}(\varphi)=\left(\frac{i}{2 \pi} \partial \bar{\partial} \varphi\right)^{m} \in \mathcal{D}^{\prime m, m}\left(\mathbb{C P}^{m}\right)
$$


When $\varphi$ is invariant under the $\mathbf{T}^{m}$ action on $\left(\mathbb{C}^{*}\right)^{m}$, then

$$
\operatorname{MA}(\varphi)=\operatorname{det}\left(\frac{1}{2 \pi} D_{\rho}^{2} \varphi\right) d \rho d \theta
$$

where $D_{\rho}^{2}$ is the real Hessian on $\mathbb{R}^{m}$.

Our results are asymptotic formulas as the degree $N \rightarrow \infty$, but with the number $f$ of monomials held fixed. For each ensemble, the limit distribution of zeros in the point case is the Monge-Ampère measure of a limit $\mathbf{T}^{m}$-independent potential, and thus the formula is of the type (11). The results are very similar for the Fubini-Study $\mathrm{SU}(m+1)$ ensemble and for general toric Gaussian measures based on inner products $G_{N}\left(\varphi, d V_{\varphi}\right)$ with $\varphi$ a toric Kähler potential. Hence we concentrate on the $\mathrm{SU}(m+1)$ case, and only briefly indicate the modifications needed for the general toric Kähler case.

Our first result concerns the ensemble with dilates of a fixed spectrum. Since the lattice points lie in $\mathbb{R}^{m}$ we use upper subscripts to index the different points in the spectrum and lower subscripts to index their coordinates. We recall that $\mathbf{E}_{N \mid S}$ refers to the expectation with respect to the conditional Gaussian measure $\gamma_{N \mid S}$.

THEOREM 1. Let $S=\left\{\lambda^{1}, \ldots, \lambda^{f}\right\}$ be a fixed spectrum consisting of $f$ lattice points in $p \Sigma$. For random m-tuples $\left(P_{1}^{N}, \ldots, P_{m}^{N}\right)$ of fewnomials in $\operatorname{Poly}(N S)$, with coefficients chosen from the $\mathrm{SU}(m+1)$ ensembles of degree $p N$, the expected distribution of zeros in $\left(\mathbb{C}^{*}\right)^{m}$ has the asymptotics

$$
N^{-m} \mathbf{E}_{N p \mid N S}\left[Z_{P_{1}^{N}, \ldots, P_{m}^{N}}\right] \rightarrow p^{m} \mathrm{MA}\left(\max _{\lambda \in S}\left[\langle\rho, \lambda\rangle-\left\langle\widehat{\lambda}^{p}, \log \widehat{\lambda}^{p}\right\rangle\right]\right)
$$

Here, $\widehat{\lambda}^{p}=\left(p-|\lambda|, \lambda_{1}, \ldots, \lambda_{m}\right)$ and $\log \widehat{\lambda}^{p}=\left(\log (p-|\lambda|), \log \lambda_{1}, \ldots, \log \lambda_{m}\right)$.

For a spectrum $S \subset p \Sigma$, we let $\mathcal{L}_{S}^{p}$ denote the Monge-Ampère potential in Theorem 1:

$$
\mathcal{L}_{S}^{p}(\rho):=\max _{\lambda \in S}\left[\langle\rho, \lambda\rangle-\left\langle\widehat{\lambda}^{p}, \log \widehat{\lambda}^{p}\right\rangle\right], \quad \rho \in \mathbb{R}^{m} .
$$

It is kind of discrete Legendre transform of the entropy function $\left\langle\widehat{\lambda}^{p}, \log \widehat{\lambda}^{p}\right\rangle$, which is the symplectic potential corresponding to the Fubini-Study Kähler potential.

We note that the expected limit distribution is a singular measure invariant under rotations of the angular variables and supported along the 0-dimensional corner set of the piecewise linear function $\mathcal{L}_{S}^{p}(\rho)$. This reflects the heuristic principle that the zeros of a fewnomial should come from its sub-fewnomials with fewnomial number $f=m+1$.

With no additional effort, we could fix the spectra separately for each polynomial in the system, and obtain:

THEOREM 2. Let $S^{1}, \ldots, S^{k}$ be fixed finite spectra consisting of lattice points in $p \Sigma$, where $1 \leq k \leq m$. For random fewnomial $k$-tuples $\left(P_{1}^{N}, \ldots, P_{m}^{N}\right)$ in $\operatorname{Poly}\left(N S^{1}\right) \times \cdots \times \operatorname{Poly}\left(N S^{k}\right)$, with coefficients chosen from the $\mathrm{SU}(m+1)$ ensembles of degree $p N$, the expected zero current in $\left(\mathbb{C}^{*}\right)^{m}$ has the asymptotics

$$
N^{-k} \mathbf{E}_{N S^{1}, \ldots, N S^{k}}\left[Z_{P_{1}^{N}, \ldots, P_{k}^{N}}\right] \rightarrow \bigwedge_{j=1}^{k}\left(\frac{i p}{2 \pi} \partial \bar{\partial} \mathcal{L}_{S_{j}}^{p}(\rho)\right) .
$$


We now state the result for fewnomial ensembles in which we randomize the spectra in the sense of (II):

Corollary 3. Let $\Delta \subset p \Sigma$ be a (fixed) Newton polytope, let $S^{1}, \ldots, S^{k}$ be random spectra contained in $\Delta$ with fewnomial number $f$, and let $P_{1}^{N}, \ldots, P_{k}^{N}$ be random fewnomial $k$-tuples $\left(P_{1}^{N}, \ldots, P_{m}^{N}\right)$ in $\operatorname{Poly}\left(N S^{1}\right) \times \cdots \times \operatorname{Poly}\left(N S^{k}\right)$, with coefficients chosen from the $\mathrm{SU}(m+1)$ ensembles of degree $p N$. Then the expected zero current in $\left(\mathbb{C}^{*}\right)^{m}$ has the asymptotics

$$
N^{-k} \mathbf{E}\left[Z_{P_{1}^{N}, \ldots, P_{k}^{N}}\right] \rightarrow \frac{1}{C(\Delta, f)^{k}}\left(\frac{i p}{2 \pi} \partial \bar{\partial} \sum_{S \in \mathcal{C}(\Delta, f)} \mathcal{L}_{S}^{p}(\rho)\right)^{k}
$$

Next, instead of dilating random spectra, we consider completely random spectra as described in (III) and we obtain:

ThEOREM 4. Let $1 \leq k \leq m$, and let $\left(P_{1}, \ldots, P_{k}\right)$ be a random system of fewnomials of fewnomial number $f$ and of degree $N$, where the spectra $S_{j}$ are chosen uniformly at random from the simplex $N \Sigma$ and the coefficients are chosen from the $\mathrm{SU}(m+1)$ ensemble. Then the expected zero current in $\left(\mathbb{C}^{*}\right)^{m}$ has the asymptotics

$$
N^{-k} \mathbf{E}_{N, f}\left[Z_{P_{1}^{N}, \ldots, P_{k}^{N}}\right] \rightarrow\left(\frac{i}{2 \pi} \partial \bar{\partial} \int_{\Sigma^{f}} \max _{j=1, \ldots, f}\left[\left\langle\rho, \lambda^{j}\right\rangle-\left\langle\widehat{\lambda^{j}}, \log \widehat{\lambda^{j}}\right\rangle\right] d \lambda^{1} \cdots d \lambda^{f}\right)^{k} .
$$

Here, $\widehat{\lambda}=\widehat{\lambda}^{1}=\left(1-|\lambda|, \lambda_{1}, \ldots, \lambda_{m}\right), d \lambda=m ! d \lambda_{1} \cdots d \lambda_{m}$.

The limit measure is thus the Monge-Ampère measure of the limit potential obtained by averaging the discrete Legendre transform $\mathcal{L}_{\left\{\lambda_{1}, \ldots, \lambda_{f}\right\}}^{1}(\rho)$ from Theorems 1 and 2 (with $p=1$ ) over all choices of points $\lambda^{1}, \ldots, \lambda^{f}$ of $\Sigma$.

We note that the averaging smooths out the corners. Indeed, we have the following more explicit formula for the expected limit distribution:

Corollary 5. Let $\left(P_{1}, \ldots, P_{k}\right)$ be the random system of Theorem 4. Then

$$
N^{-k} \mathbf{E}_{N, f}\left[Z_{P_{1}^{N}, \ldots, P_{k}^{N}}\right] \rightarrow\left\{\omega_{\mathrm{FS}}-\frac{i}{2 \pi} \int_{0}^{\infty} \partial \bar{\partial}\left(\left[1-D_{b}(t ; \rho)\right]^{f}\right) d t\right\}^{k}
$$

where

$$
D_{b}(t ; \rho)=m ! \operatorname{Vol}\left(\left\{\lambda \in \Sigma:\langle\widehat{\lambda}, \log \widehat{\lambda}\rangle-\langle\rho, \lambda\rangle+\log \left(1+\left|e^{\rho}\right|\right) \leq t\right\}\right) .
$$

Here, $\omega_{\mathrm{FS}}=\frac{i}{2 \pi} \partial \bar{\partial} \log \left(1+\left|e^{\rho}\right|\right)$ is the Fubini-Study Kähler form on $\left(\mathbb{C}^{*}\right)^{m} \subset \mathbb{C P}^{m}$. The quantity $D_{b}(t ; \rho)$ is the distribution function for the pointwise logarithmic decay rate $b_{\lambda}(\rho)$ of the monomials $\varphi_{N \lambda}$ (see $\S 2.3$ ), regarded as a random variable (with parameter $\rho$ ) on $\Sigma$. Note that the integral in Corollary 5 is actually over a bounded interval.

We can also generalize Theorem 4 to the ensemble (IV):

TheOREM 6. Let $1 \leq k \leq m$, let $\Delta_{1} \subset p_{1} \Sigma, \ldots, \Delta_{k} \subset p_{k} \Sigma$ be Newton polytopes and let $\left(P_{1}, \ldots, P_{k}\right)$ be a random system of fewnomials of fewnomial numbers $f_{1}, \ldots, f_{k}$ respectively, where the spectra $S_{j}$ are chosen uniformly at random from the simplices $N \Delta_{j}$ and the coefficients are chosen from the $\mathrm{SU}(m+1)$ ensemble. Then the expected zero current in $\left(\mathbb{C}^{*}\right)^{m}$ 
has the asymptotics

$N^{-m} \mathbf{E}\left[Z_{P_{1}^{N}, \ldots, P_{k}^{N}}\right] \rightarrow \bigwedge_{j=1}^{k}\left(\frac{i}{2 \pi} \frac{p_{j}}{\operatorname{Vol}\left(\Delta_{j}\right)^{f_{j}}} \partial \bar{\partial} \int_{\Delta_{j}^{f_{j}}} \max _{l=1, \ldots, f_{j}}\left[\left\langle\rho, \lambda^{l}\right\rangle-\left\langle\widehat{\lambda^{l}}, \log \widehat{\lambda^{l}}\right\rangle\right] d \lambda^{1} \cdots d \lambda^{f_{j}}\right)$.

Here, $\widehat{\lambda}=\widehat{\lambda}^{1}=\left(1-|\lambda|, \lambda_{1}, \ldots, \lambda_{m}\right), d \lambda=d \lambda_{1} \cdots d \lambda_{m}$.

The key analytical ingredient in the proofs of these results is an asymptotic formula for the expected mass density of the above systems of random polynomials as $N \rightarrow \infty$. It is given by the conditional Szegö kernel $\Pi_{N, Q \mid S}$ with respect to the norm $Q_{G_{N}(\varphi, \nu)}(6)$ and spectrum $S$, i.e. the kernel of the orthogonal projection (Szegö kernel) onto the subspace of polynomials under consideration:

$$
\mathbf{E}_{N, Q \mid S}\left(|P(z)|_{N \varphi}^{2}\right)=\sum_{\alpha \in S} \frac{\left|\chi_{\alpha}(z)\right|_{N \varphi}^{2}}{\left\|\chi_{\alpha}\right\|_{Q}^{2}}=\Pi_{N, Q \mid S}(z, z) .
$$

Thus, the results depend on the asymptotics of the Szegö kernels $\Pi_{N, Q \mid S}(z, z)$.

0.4. More general toric weights. We briefly indicate the generalization when the $\mathrm{SU}(m+$ 1) (Fubini-Study) inner product on $\operatorname{Poly}(N)$ is replaced by $G_{N}\left(\varphi, d V_{\varphi}\right)$ for a general toric Kähler potential $\varphi$.

The polytope $P$ of the toric variety is defined by a set of linear inequalities

$$
\ell_{r}(x):=\left\langle x, v_{r}\right\rangle-\lambda_{r} \geq 0, r=1, \ldots, d,
$$

where $v_{r}$ is a primitive element of the lattice and inward-pointing normal to the $r$-th $(m-1)$ dimensional facet $F_{r}=\left\{\ell_{r}=0\right\}$ of $P$.

A $\mathbf{T}^{m}$-invariant Kähler potential on $\left(\mathbb{C}^{*}\right)^{m}$ defines a real convex function on $\rho \in \mathbb{R}^{m}$. Its Legendre transform

$$
u_{\varphi}(x):=\mathcal{L} \varphi(x):=\sup _{\rho}\left(\langle x, \rho\rangle-\varphi\left(e^{\rho}\right)\right)
$$

is the symplectic potential $u_{\varphi}$. Equivalently, for $x \in P$, there is a unique $\rho$ such that $\nabla_{\rho} \varphi=x$, and $u_{\varphi}(x)=\left\langle x, \rho_{x}\right\rangle-\varphi\left(\rho_{x}\right)$. In the Fubini-Study case, $P=\Sigma, \varphi=\log \left(1+e^{\rho}\right)$, and $u_{F S}(x)=\sum_{k} \ell_{k}(x) \log \ell_{k}(x)$ where $\ell_{k}(x)=x_{k}$ for $k=1, \ldots, m$ and $\ell_{m+1}(x)=1-|x|$ where $|x|=x_{1}+\cdots+x_{m}$ (in multi-index notation on $\mathbb{R}^{m}$ ). Thus,

$$
u_{F S}(\lambda)=\langle\widehat{\lambda}, \log \widehat{\lambda}\rangle \text {. }
$$

The Kähler potential is the Legendre transform $\mathcal{L} u_{\varphi}(\rho)$ of its symplectic potential. If we allowed all possible spectra in the ensemble (hence not a fewnomial ensemble), the discrete Legendre transforms with respect to $f$-element subsets would converge to the usual Legendre transform and the potential in (12) would become $\varphi$. Thus, the impact of the restriction to $f$ monomials is that in place of the Legendre transform we have an average of discrete Legendre transforms.

As this indicates, the result for a general toric Kähler Gaussian ensemble for $\mathbb{C P}^{m}$ and polytope $\Sigma$, defined by $G_{N}\left(\varphi, d V_{\varphi}\right)$, is the following: 
TheOREm 7. Consider the ensembles of type (III) as in Theorem 4, but with Gaussian measures induced by the inner product $G_{N}\left(\varphi, d V_{\varphi}\right)$ corresponding to a toric Kähler potential on $\mathbb{C P}^{m}$. Then the expected distribution of zeros in $\left(\mathbb{C}^{*}\right)^{m}$ has the asymptotics

$$
N^{-m} \mathbf{E}\left[Z_{P_{1}^{N}, \ldots, P_{k}^{N}}\right] \rightarrow\left(\frac{i}{2 \pi} \partial \bar{\partial} \int_{\Sigma^{f} j=1, \ldots, f}\left[\left\langle\rho, \lambda^{j}\right\rangle-u_{\varphi}(\lambda)\right] d \lambda^{1} \cdots d \lambda^{f}\right)^{k} .
$$

The proof is almost the same as for the Fubini-Study case and is indicated in $\S 4$. In $\S 5$, we also indicate the modifications in the case of the fewnomial Kac-Hammersley ensemble.

\section{Preliminaries}

In this section, we review the relation between inner products on spaces of polynomials and associated Gaussian measures on the space. The inner products implicitly involve a choice of Kähler metric on $\mathbb{C P}^{m}$. The associated Kähler potential determines the shape of the modulus of each monomial and its concentration properties.

We may identify a not necessarily homogeneous polynomial $f$ on $\mathbb{C}^{m}$ of degree $\leq N$ by its homogenization as a polynomial

$$
F\left(\zeta_{0}, \ldots, \zeta_{m}\right)=\sum_{|\lambda|=N} C_{\lambda} \zeta^{\lambda} \quad\left(\zeta^{\lambda}=\zeta_{0}^{\lambda_{0}} \cdots \zeta_{m}^{\lambda_{m}}\right)
$$

of degree $N$ in $m+1$ variables, where

$$
f\left(z_{1}, \ldots, z_{m}\right)=F\left(1, z_{1}, \ldots, z_{m}\right)=\sum_{|\alpha| \leq N} c_{\alpha} z^{\alpha} \quad\left(z^{\alpha}=z_{1}^{\alpha_{1}} \cdots z_{m}^{\alpha_{m}}\right),
$$

where $c_{\alpha}=C_{\hat{\alpha}^{N}}, \hat{\alpha}^{N}=\left(N-|\alpha|, \alpha_{1}, \ldots, \alpha_{m}\right),|\alpha|=\sum_{j=1}^{m} \alpha_{j}$. Homogeneous polynomials of degree $N$ on $\mathbb{C}^{m+1}$ are equivalent to holomorphic sections $H^{0}\left(\mathbb{C P}^{m}, \mathcal{O}(N)\right)$ of the $N$ th power of the hyperplane section bundle. This geometric identification is useful in interpreting the concentration properties of monomials in terms of curvature.

We let $e_{0} \in H^{0}\left(\mathbb{C P}^{m} \mathcal{O}(1)\right)$ be the degree 1 polynomial $e_{0}\left(\zeta_{0}, \ldots, \zeta_{m}\right)=\zeta_{0}$. Then $e_{0}$ is a local frame over the affine chart $U_{0}=\left\{\zeta_{0} \neq 0\right\} \approx \mathbb{C}^{m}$. We fix a Hermitian metric $h$ on $\mathcal{O}(1)$. In the local frame $e_{0}$, the metric has the local expression $h=e^{-\varphi}$, where $\varphi$ is known as the Kähler potential. The Kähler form is denoted by $\omega_{\varphi}=\frac{i}{2 \pi} \partial \bar{\partial} \varphi$.

We define the inner product on $\operatorname{Poly}(N \Sigma)$ :

$$
\langle f, \bar{g}\rangle_{h}=\frac{1}{m !} \int_{\mathbb{C}^{m}} f(z) \overline{g(z)} e^{-N \varphi(z)} \omega_{\varphi}^{m}(z), \quad f, g \in \operatorname{Poly}(N \Sigma) .
$$

The inner product is determined by the matrix of inner products on the distinguished basis of monomials $\chi_{\alpha}$. All of our inner products are $\mathbf{T}^{m}$-invariant and hence the monomials are automatically orthogonal. The inner products are then determined by the norming constants (6), specifically,

$$
Q(\alpha)=Q_{G_{N}\left(\varphi, d V_{\varphi}\right)}(\alpha)=\frac{1}{m !} \int_{\mathbb{C}^{m}}\left|z^{\alpha}\right|^{2} e^{-N \varphi(z)} \omega_{\varphi}^{m}(z) .
$$

The inner product induces a Gaussian measure $\gamma_{h}$ on any subspace $\mathcal{S} \subset \operatorname{Poly}(N \Sigma)$. Again assuming that the monomials are orthogonal, the basis $(7)$ is $\langle,\rangle_{h}$ orthonormal and we may 
write any polynomial in the form

$$
P_{N}=\sum_{\alpha \in N \Sigma} c_{\alpha} \varphi_{\alpha}
$$

The associated Gaussian measure is defined by the condition that the coefficients of this orthonormal expansion are independent complex normal random variables.

The Szegö kernel (or weighted Bergman kernel) for the line bundle $\mathcal{O}(N)$ with metric $h^{N}=e^{-N \varphi}$ is given over $\mathbb{C}^{m}$ by

$$
\Pi_{N, Q}(z, w)=e^{-N \varphi} \sum_{\alpha \in N \Sigma} \varphi_{\alpha}^{N}(z) \overline{\varphi_{\alpha}^{N}(w)}
$$

It is the kernel for the orthogonal projection from $L^{2}(X) \rightarrow H^{0}\left(\mathbb{C} \mathbb{P}^{m}, \mathcal{O}(N)\right)$, where $X \rightarrow$ $\mathbb{C P}^{m}$ is the unit circle bundle in $\left(L^{*}, h^{*}\right)$ with fibers $X_{z}=\left\{e^{-\varphi+i \theta}\left(1, z_{0}, \ldots, z_{m}\right): \theta \in \mathbb{R}\right\}$ over points $z \in \mathbb{C}^{m}$; see [SZ1]. For spectra $S \subset \mathbb{Z}^{m} \cap N \Sigma$, then the kernel for the orthogonal projection $L^{2}(X) \rightarrow \operatorname{Poly}(S)$ is the conditional weighted Bergman kernel given by

$$
\Pi_{N, Q \mid S}(z, w)=e^{-N \varphi} \sum_{\alpha \in S} \varphi_{\alpha}^{N}(z) \overline{\varphi_{\alpha}^{N}(w)} .
$$

1.1. The $\mathrm{SU}(m+1)$-ensembles. This is the Gaussian ensemble defined by the inner product arising from the Fubini-Study metric $\varphi=\log \left(1+|z|^{2}\right)$. Then $\omega_{\mathrm{FS}}=\frac{i}{2 \pi} \partial \bar{\partial} \log \left(1+\|z\|^{2}\right)$ is the Fubini-Study Kähler form on $\mathbb{C}^{m} \subset \mathbb{C P}^{m}$ and

$$
\langle f, \bar{g}\rangle=\frac{1}{m !} \int_{\mathbb{C}^{m}} \frac{f(z) \overline{g(z)}}{\left(1+\|z\|^{2}\right)^{N}} \omega_{\mathrm{FS}}^{m}(z), \quad f, g \in \operatorname{Poly}(N \Sigma), .
$$

The norming constants for the inner product (14) are:

$$
\left\|\chi_{\alpha}\right\|=\sqrt{\left\langle\chi_{\alpha}, \chi_{\alpha}\right\rangle}=\left[\frac{N !}{(N+m) !\left(\begin{array}{c}
N \\
\alpha
\end{array}\right)}\right]^{\frac{1}{2}}, \quad\left(\begin{array}{c}
N \\
\alpha
\end{array}\right):=\frac{N !}{(N-|\alpha|) ! \alpha_{1} ! \cdots \alpha_{m} !} .
$$

Thus we have an orthonormal basis for $\operatorname{Poly}(N \Sigma)$ given by the monomials

$$
m_{\alpha}^{N}:=\frac{1}{\left\|\chi_{\alpha}\right\|} \chi_{\alpha}=\sqrt{\frac{(N+m) !}{N !}\left(\begin{array}{c}
N \\
\alpha
\end{array}\right)} \chi_{\alpha}, \quad|\alpha| \leq N .
$$

In this case, the circle bundle $X$ is the unit sphere $S^{2 m+1} \subset \mathbb{C}^{m+1}$. We now regard the sections of $H^{0}\left(\mathbb{C P}^{m}, \mathcal{O}(N)\right)$ as homogeneous polynomials restricted to $X=S^{2 m+1}$. By identifying the point $z \in\left(\mathbb{C}^{*}\right)^{m}$ with the lift $x=\frac{1}{\left(1+\|z\|^{2}\right)^{1 / 2}}\left(1, z_{1}, \ldots, z_{m}\right) \in S^{2 m+1}$, we may write the homogenized monomials on $S^{2 m+1}$ in affine coordinates $\left(z_{1}, \ldots, z_{m}\right)$ as

$$
\widehat{\chi}_{\alpha}^{N}(z)=\frac{z^{\alpha}}{\left(1+\|z\|^{2}\right)^{N / 2}} .
$$

The corresponding $L^{2}$ normalized monomials are then:

$$
\widehat{m}_{\alpha}^{N}(z):=\sqrt{\frac{(N+m) !}{N !}\left(\begin{array}{c}
N \\
\alpha
\end{array}\right)} \frac{z^{\alpha}}{\left(1+\|z\|^{2}\right)^{N / 2}}, \quad|\alpha| \leq N .
$$


In short, the $\mathrm{SU}(m+1)$ ensemble of random polynomials of degree $N$ consists of polynomials of the form

$$
\sum_{|\alpha| \leq N} c_{\alpha} \sqrt{\frac{(N+m) !}{N !}\left(\begin{array}{c}
N \\
\alpha
\end{array}\right)} \frac{z^{\alpha}}{\left(1+\|z\|^{2}\right)^{N / 2}},
$$

where $c_{\alpha}$ are independent complex normal variables of mean zero and variance one.

Specializing (17) to the Fubini-Study metric, we have the following definition (where we omit the subscript $Q$ indicating the norming constants):

Definition: Let $S \subset \mathbb{Z}^{m} \cap N \Sigma$. The conditional Fubini-Study Szegö kernel $\Pi_{N \mid S}$ is the kernel for the orthogonal projection to $\operatorname{Poly}(S)$ with respect to the induced Fubini-Study inner product:

$$
\Pi_{N \mid S}(x, y)=\sum_{\alpha \in S} \frac{1}{\left\|\chi_{\alpha}\right\|^{2}} \widehat{\chi}_{\alpha}^{N}(x) \overline{\widehat{\chi}_{\alpha}^{N}(y)}=\frac{(N+m) !}{N !} \sum_{\alpha \in P} \widehat{m}_{\alpha}^{N}(x) \overline{\widehat{m}_{\alpha}^{N}(y)} .
$$

The conditional Szegö kernel can be written explicitly on $\mathbb{C}^{m}$ as

$$
\Pi_{N \mid S}(z, w)=\frac{(N+m) !}{N !} \frac{\sum_{\alpha \in S}\left(\begin{array}{l}
N \\
\alpha
\end{array}\right) z^{\alpha} \bar{w}^{\alpha}}{\left(1+\|z\|^{2}\right)^{N / 2}\left(1+\|w\|^{2}\right)^{N / 2}} .
$$

It is the two-point function for the conditional Gaussian ensemble $\operatorname{Poly}(S) \subset \operatorname{Poly}(N)$.

The full Fubini-Study Szegö kernel is given by

$$
\begin{aligned}
\Pi_{N}(z, w) & =\frac{(N+m) !}{N !} \sum_{|\alpha| \leq N} \widehat{\chi}_{\alpha}^{N}(z) \overline{\widehat{\chi}_{\alpha}^{N}(w)}=\frac{(N+m) !}{N !} \frac{\sum_{|\alpha| \leq N}\left(\begin{array}{c}
N \\
\alpha
\end{array}\right) z^{\alpha} \bar{w}^{\alpha}}{\left(1+\|z\|^{2}\right)^{N / 2}\left(1+\|w\|^{2}\right)^{N / 2}}(26) \\
& =\frac{(N+m) !}{N !}\left[\frac{1+\langle z, \bar{w}\rangle}{\left(1+\|z\|^{2}\right)^{1 / 2}\left(1+\|w\|^{2}\right)^{1 / 2}}\right]^{N}
\end{aligned}
$$

\section{Fewnomial Ensembles}

2.1. Precise definitions of random fewnomials. We now define more precisely the ensembles which allow for any fewnomial system. We fix the degree $N$, and first consider the case of one random fewnomial. We specify a set of lattice points by its characteristic function

$$
\sigma: N \Sigma \cap \mathbb{N}^{m} \rightarrow\{0,1\}
$$

which may be regarded as an occupation number, designating whether a lattice point is occupied $(\sigma(\alpha)=1)$ or unoccupied $\left(\sigma(\alpha)=0\right.$. We denote by $|\sigma|=\sum_{\alpha \in N \Sigma} \sigma(\alpha)$ the number of elements in the set, and by $\operatorname{Supp} \sigma=\{\alpha: \sigma(\alpha)=1\}$ the support of $\sigma$. We put:

$$
\mathcal{C}_{N, f}=\left\{\sigma: N \Sigma \cap \mathbb{N}^{m} \rightarrow\{0,1\} \text { such that }|\sigma|=f\right\},
$$

and we denote the number of such subsets by

$$
C(N, f)=\left|\mathcal{C}_{N, f}\right|=\left(\begin{array}{c}
N+m \\
m \\
f
\end{array}\right)=\frac{1}{(m !)^{f} f !} N^{m f}+O\left(N^{m f-1}\right) .
$$

A polynomial with (at most) $f$ non-zero terms can then be written in the form:

$$
P_{\sigma, c}(z)=\sum_{\alpha \in N \Sigma} \sigma(\alpha) c_{\alpha} z^{\alpha}, \quad|\sigma|=f .
$$


Thus the space of random fewnomials is given by:

$$
\mathcal{F}_{N, f}=\left\{(\sigma, P) \in \mathcal{C}_{N, f} \times \operatorname{Poly}(N): P \in \operatorname{Poly}(\operatorname{Supp} \sigma)\right\} .
$$

There is a natural projection $\pi: \mathcal{F}_{N, f} \rightarrow \mathcal{C}_{N, f}$ taking $(\sigma, P) \rightarrow \sigma$ and the 'fiber' of this projection is $\operatorname{Poly}(\operatorname{Supp} \sigma)$. The set of fewnomial systems of $m$ polynomials in $m$ variables with fewnomial numbers $\left(f_{1}, \ldots, f_{m}\right)$ is then given by

$$
\mathcal{F}_{N,\left(f_{1}, \ldots, f_{m}\right)}:=\mathcal{F}_{N, f_{1}} \times \cdots \times \mathcal{F}_{N, f_{m}} .
$$

It is also natural to consider fewnomials with spectra contained in a given Newton polytope. We therefore fix a convex lattice polytope $\Delta \subset p \Sigma$ (for some $p$ ) and replace $\Sigma$ everywhere by $\Delta$. Thus, we define

$$
\mathcal{C}_{N, f, \Delta}=\left\{\sigma: N \Delta \cap \mathbb{N}^{m} \rightarrow\{0,1\} \text { such that }|\sigma|=f\right\},
$$

and

$$
\mathcal{F}_{N, f, \Delta} \subset \mathcal{C}_{N, f, \Delta} \times \operatorname{Poly}(N)=\{(\sigma, P): \operatorname{Supp}(\sigma) \subset N \Delta, P \in \operatorname{Poly}(\operatorname{Supp} \sigma)\} . .
$$

Similarly, we define $\mathcal{F}_{N,\left(f_{1}, \ldots, f_{m}\right), \Delta_{1}, \ldots, \Delta_{m}}$ for systems.

We now induce probability measures on $\mathcal{F}_{N, f}$ and $\mathcal{F}_{N, f, \Delta}$, by regarding them as 'fibering' over $\mathcal{C}(N, f)$, by putting counting measure on $\mathcal{C}_{N, f}$ and by putting the conditional measures $d \gamma_{N \mid \text { supp } \sigma}$ on the 'fibers'.

Definition: The ensemble of random $\mathrm{SU}(m+1)$ fewnomials of degree $N$ and fewnomial number $f$ is the space $\mathcal{F}_{N, f}$ endowed with the probability measure $d \mu_{N, f}$ defined by

$$
\int_{\mathcal{F}_{N, f}} g(S, P) d \mu_{N, f}(S, P):=\frac{1}{C(N, f)} \sum_{S \in \mathcal{C}(N, f)} \int_{\operatorname{Poly}(S)} g(S, p) d \gamma_{N \mid S}(P) .
$$

In other words, $d \mu_{N, f}$ is defined by putting counting measure on $\mathcal{C}_{N, f}$ and by putting the conditional measures $d \gamma_{N \mid S}$ (given by (9) with $\varphi_{\alpha}=\widehat{m}_{\alpha}^{N}$ ) on the 'fibers' of $\pi$.

We then put the product measures

$$
d \mu_{N, f_{1}, \ldots, f_{k}}=d \mu_{N, f_{1}} \times \cdots \times d \mu_{N, f_{k}}
$$

on the space $\mathcal{F}_{N,\left(f_{1}, \ldots, f_{m}\right)}$ of systems.

We define the measure $d \mu_{N, f, \Delta}$ on $\mathcal{F}_{N, f, \Delta}$ and on the associated systems analogously. Similarly we define the measures $d \mu_{N, f}^{\varphi, \nu}$ and $d \mu_{N, f}^{K H}$ for the general toric and Kac-Hammersley ensembles, respectively.

2.2. Expected zeros of fewnomial ensembles. We recall the probabilistic PoincaréLelong formula (see for example,[SZ1, SZ3]):

Proposition 2.1. Let $(L, h)$ be a Hermitian line bundle on a compact Kähler manifold $M$. Let $\mathcal{S}$ be a subspace of $H^{0}(M, L)$ endowed with a Hermitian inner product and we let $\gamma$ be the induced Gaussian probability measure on $\mathcal{S}$. Then the expected zero current of a random section $s \in \mathcal{S}$ is given by

$$
\mathbf{E}_{\gamma}\left(Z_{s}\right)=\frac{\sqrt{-1}}{2 \pi} \partial \bar{\partial} \log \Pi_{\mathcal{S}}(z, z)+c_{1}(L, h) .
$$


If $\mathcal{S}_{j}$ is a base-point-free linear system with Gaussian probability measure $\gamma_{j}$, for $1 \leq j \leq k$ (where $1 \leq k \leq m$ ), then the expected value of the simultaneous zero current of $k$ independent random sections $s_{1} \in \mathcal{S}_{1}, \ldots, s_{k} \in \mathcal{S}_{k}$ is given by

$$
\mathbf{E}_{\gamma_{1}, \ldots, \gamma_{k}}\left(Z_{s_{1}, \ldots, s_{k}}\right)=\bigwedge_{j=1}^{k}\left(\frac{\sqrt{-1}}{2 \pi} \partial \bar{\partial} \log \Pi_{\mathcal{S}_{j}}(z, z)+c_{1}(L, h)\right),
$$

which is a smooth form.

Applying Proposition 2.1 to a fewnomial system $\mathcal{S}=\operatorname{Poly}(S)$, we have

Proposition 2.2. Let $S_{1}, \ldots, S_{k}$ be finite subsets of $N \Sigma \cap \mathbb{Z}^{m}$. Then the expected zero current in $\left(\mathbb{C}^{*}\right)^{m}$ of $k$ random fewnomials $P_{1} \in \operatorname{Poly}\left(S_{1}\right), \ldots, P_{k} \in S_{k}$ is given by the smooth form

$$
\mathbf{E}_{N \mid S_{1}, \ldots, S_{k}} Z_{P_{1}, \ldots, P_{k}}=\bigwedge_{j=1}^{k}\left(\frac{\sqrt{-1}}{2 \pi} \partial \bar{\partial} \log \Pi_{S_{j}}(z, z)+\frac{N}{\pi} \omega_{\mathrm{FS}}\right) .
$$

Proof. We recall that the base point locus of a suspace $\mathcal{S} \subset \operatorname{Poly}(N)$ is the set of points at which $p(z)=0, \forall p \in \mathcal{S}$. Since a monomial $z_{1}^{\alpha_{1}} \cdots z_{m}^{\alpha_{m}}$ vanishes if and only if $z_{j}=0$ for some $j$ such that $\alpha_{j}>0$, the base locus of $\operatorname{Poly}(S)$ is always contained in the coordinate hyperplances $\bigcup_{j=1}^{m}\left\{z_{j}=0\right\}$. Applying Proposition 2.1 to $\left(\mathbb{C}^{*}\right)^{m} \subset \mathbb{C P}^{m}$, we obtain the result.

Corollary 2.3. The expected zero current in $\left(\mathbb{C}^{*}\right)^{m}$ of a system of $k$ random fewnomials of degree $\leq N$ with fewnomial number $f$ is given by

$$
\mathbf{E}_{N, f} Z_{P_{1}, \ldots, P_{k}}=\left[\frac{1}{C(N, f)} \sum_{\sigma \in \mathcal{C}_{N, f}}\left(\frac{\sqrt{-1}}{2 \pi} \partial \bar{\partial} \log \Pi_{N \mid \operatorname{Supp} \sigma}(z, z)+\frac{N}{\pi} \omega_{\mathrm{FS}}\right)\right]^{k} .
$$

where $C(N, f)$ is given by $(30)$.

2.3. Mass asymptotics and fewnomial Szegö kernels. We now give the asymptotics of the Szegö kernels $\Pi_{N \mid S}$ We need joint asymptotics in $N$ and $S$ (leaving the fewnomial number $f=|S|$ fixed). We begin with the dilated fixed spectra system (I).

A special case of Theorem 4.1 in [SZ1] on the mass asymptotics for polynomials with spectra in dilates of a Newton polytope $P$ is where $P=\{\beta\}$ is a single lattice point in $p \Sigma$. In this case

$$
\Pi_{N p \mid N\{\beta\}}(z, z)=\left|\widehat{m}_{N \beta}^{N p}\right|^{2}=N^{\frac{m}{2}} e^{-N b_{\beta}^{p}(z)}\left[c_{0}+c_{1} N^{-1}+c_{2} N^{-2}+\cdots\right],
$$

where

$$
b_{\beta}^{p}(z)=\sum_{j=0}^{m} \beta_{j} \log \frac{\beta_{j}}{p}-\log \frac{\left|z^{\beta}\right|^{2}}{\left(1+\|z\|^{2}\right)^{p}} \quad\left(\beta_{0}=p-|\beta|\right) .
$$

In (37), we can let $\beta$ be any point in the interior of $p \Sigma$. We also write $b_{x}=b_{x}^{1}$, for arbitrary (not necessarily integral) $x \in \Sigma$ :

$$
b_{x}(z)=\sum_{j=0}^{m} x_{j} \log x_{j}-\sum_{j=1}^{m} x_{j} \log \left|z_{j}\right|^{2}+\log \left(1+\|z\|^{2}\right) \quad\left(x_{0}=1-|x|\right) .
$$


The first term is the symplectic potential for the Fubini-Study metric, i.e. the Legendre transform of the open orbit Kähler potential (see §4) We now give a precise estimate for the joint asymptotics of (36) using Stirling's formula. A similar analysis was done in dimension one in [SoZ1] and in Lemma 6.2 of [SoZ3]. The kernel $(36)$ is denoted $\mathcal{P}_{h^{N}}(\alpha, z)$ in [SoZ2] and is analyzed for general toric varieties in Section 6 of that article. Since it is elementary we give a self-contained proof in the case of $\mathrm{SU}(m+1)$ polynomials (i.e., for the Fubini-Study metric).

LEMma 2.4. There exists positive constants $C_{m}$ depending only on $m$ such that for all $\alpha \in$ $(N \Sigma)^{\circ} \cap \mathbb{Z}^{m}$, we have

$$
\log \left|\widehat{m}_{\alpha}^{N}\right|^{2}=-N b_{\alpha / N}+\frac{m}{2} \log N-\frac{1}{2} \sum_{j=0}^{m} \log \left(\frac{\alpha_{j}}{N}\right)+R(\alpha, N, m),
$$

where $|R(\alpha, N, m)| \leq C_{m}$.

Proof. Let $x=\alpha / N$. Recalling (22), it suffices to show that

$\log \left[\frac{(N+m) !}{N !}\left(\begin{array}{c}N \\ \alpha\end{array}\right)\right]=-\sum_{j=0}^{m}\left(N x_{j}+\frac{1}{2}\right) \log x_{j}+\frac{m}{2} \log N+R(\alpha, N, m) \quad\left(\alpha_{0}=N-|\alpha|\right)$.

Using Stirling's formula

$$
n !=\sqrt{2 \pi} n^{n+1 / 2} e^{-n+\varepsilon_{n}}, \quad \text { where } \frac{1}{12 n+1}<\varepsilon_{n}<\frac{1}{12 n},
$$

we obtain

$$
\begin{aligned}
\log \left[\frac{(N+m) !}{N !}\left(\begin{array}{l}
N \\
\alpha
\end{array}\right)\right] \\
\quad=\sum_{j=1}^{m} \log (N+j)-\frac{m}{2} \log (2 \pi)+\left(N+\frac{1}{2}\right) \log N-\sum_{j=0}^{m}\left(\alpha_{j}+\frac{1}{2}\right) \log \alpha_{j}+\varepsilon_{N}-\sum_{j=0}^{m} \varepsilon_{\alpha_{j}} \\
\quad=\frac{m}{2} \log N-\sum_{j=0}^{m}\left(N x_{j}+\frac{1}{2}\right) \log x_{j}+R
\end{aligned}
$$

where

$$
R=\sum_{j=1}^{m} \log (1+j / N)-\frac{m}{2} \log (2 \pi)+\varepsilon_{N}-\sum \varepsilon_{\alpha_{j}}
$$

Thus

$$
|R| \leq \sum_{j=1}^{m} \log (1+j)+\frac{m}{2} \log (2 \pi)+\frac{m+1}{12}
$$

LEMmA 2.5. There exists positive constants $C_{m}^{\prime}$ such that

$$
-N b_{\alpha / N}+\frac{m}{2} \log N-C_{m}^{\prime} \leq \log \left|\widehat{m}_{\alpha}^{N}\right|^{2} \leq-N b_{\alpha / N}+m \log N+C_{m}^{\prime},
$$

for all $\alpha \in(N \Sigma) \cap \mathbb{Z}^{m}$. 
Proof. We first suppose that $\alpha \in I_{N}:=(N \Sigma)^{\circ} \cap \mathbb{Z}^{m}$. The lower bound is an immediate consequence of Lemma 2.4. If $I_{N} \neq \emptyset$, then $N \geq m+1$ and the maximum value of the convex function $\alpha \mapsto-\sum_{j=0}^{m} \log \left(\frac{\alpha_{j}}{N}\right)$ on $I_{N}$ is attained on the vertices of $I_{N}$. Thus

$$
-\frac{1}{2} \sum_{j=0}^{m} \log \left(\frac{\alpha_{j}}{N}\right) \leq \frac{m}{2} \log N+\frac{1}{2} \log \frac{N}{N-m} \leq \frac{m}{2} \log N+\frac{1}{2} \log (m+1),
$$

and the upper bound follows from Lemma 2.4.

Now suppose that $\alpha \in \partial(N \Sigma) \cap \mathbb{Z}^{m}$. By a permutation of homogenous coordinates, we can assume without loss of generality that $\alpha=\left(\alpha_{0}, \ldots, \alpha_{k}, 0, \ldots, 0\right)$ where $\alpha_{j} \geq 1$ for $0 \leq j \leq k$. Let $\alpha^{\prime}=\left(\alpha_{1}, \ldots, \alpha_{k}\right), z^{\prime}=\left(z_{1}, \ldots, z_{k}\right)$. We note that

$$
b_{\alpha^{\prime} / N}\left(z^{\prime}\right)-b_{\alpha / N}(z)=\log \left(1+\left\|z^{\prime}\right\|^{2}\right)-\log \left(1+\|z\|^{2}\right) \text {. }
$$

By the lower bound proved above for the monomial $\widehat{m}_{\alpha^{\prime}}^{N}$ on $\left(\mathbb{C}^{*}\right)^{k}$, we have

$$
\begin{aligned}
\log \left|\widehat{m}_{\alpha}^{N}(z)\right|^{2} & =\log \left|\widehat{m}_{\alpha^{\prime}}^{N}\left(z^{\prime}\right)\right|^{2}+\log \left[\frac{(N+m) !}{(N+k) !}\right]+N \log \left[\frac{1+\left\|z^{\prime}\right\|^{2}}{1+\|z\|^{2}}\right] \\
& \geq-N b_{\alpha^{\prime} / N}\left(z^{\prime}\right)+\frac{k}{2} \log N-C_{k}^{\prime}+(m-k) \log N+N \log \left[\frac{1+\left\|z^{\prime}\right\|^{2}}{1+\|z\|^{2}}\right] \\
& =-N b_{\alpha / N}(z)+\left(m-\frac{k}{2}\right) \log N-C_{k}^{\prime},
\end{aligned}
$$

which yields the desired lower bound when $\alpha$ is in the boundary of $N \Sigma$.

On the other hand, by the upper bound for the monomial $\widehat{m}_{\alpha^{\prime}}^{N}$, we have

$$
\begin{aligned}
\log \left|\widehat{m}_{\alpha}^{N}(z)\right|^{2} & =\log \left|\widehat{m}_{\alpha^{\prime}}^{N}\left(z^{\prime}\right)\right|^{2}+\log \left[\frac{(N+m) !}{(N+k) !}\right]+N \log \left[\frac{1+\left\|z^{\prime}\right\|^{2}}{1+\|z\|^{2}}\right] \\
& \leq-N b_{\alpha^{\prime} / N}\left(z^{\prime}\right)+k \log N+C_{k}^{\prime}+(m-k)\left(\log N+\frac{m}{N}\right)+N \log \left[\frac{1+\left\|z^{\prime}\right\|^{2}}{1+\|z\|^{2}}\right] \\
& =-N b_{\alpha / N}(z)+m \log N+C_{k}^{\prime}+\frac{m^{2}-k m}{N},
\end{aligned}
$$

which gives the desired upper bound.

2.4. Proof of Theorems $\mathbf{1}$ and 2. These theorems are consequences of the following convergence result:

Lemma 2.6. Let $m, f, p$ be positive integers. Then

$$
\frac{1}{N} \log \Pi_{N p \mid N S}(z, z) \rightarrow-p \min _{1 \leq j \leq f}\left\{b_{\lambda^{j} / p}(z)\right\}
$$

uniformly for $z \in\left(\mathbb{C}^{*}\right)^{m}, S \in \mathcal{C}(p, f)$.

Proof. Let $S=\left\{\lambda^{1}, \ldots, \lambda^{f}\right\} \subset p \Sigma$, and recall that

$$
\Pi_{N p \mid N S}(z, z)=\sum_{j=1}^{f}\left|\widehat{m}_{N \lambda^{j}}^{N p}(z)\right|^{2}
$$


By Lemma 2.5, we have

$$
\begin{array}{r}
\max _{j}\left\{-N p b_{\lambda^{j} / p}\right\}-C_{m} \leq \max _{j}\left\{\log \left|\widehat{m}_{N \lambda^{j}}^{N p}\right|^{2}\right\} \leq \log \prod_{N p \mid N S} \leq \max _{j}\left\{\log \left|\widehat{m}_{N \lambda^{j}}^{N p}\right|^{2}\right\}+\log f \\
\leq \max _{j}\left\{-N p b_{\lambda^{j} / N}\right\}+m \log (N p)+C_{m}^{\prime}+\log f .
\end{array}
$$

Dividing by $N$, the conclusion follows.

Remark: Lemma 2.6 is a special case of the generalization (with a stronger uniformity result) of Proposition 4.2 in [SZ2] to nonconvex polytopes [unpublished]. In the case where $S$ is one point, an analysis of the full (i.e. not just logarithmic) asymptotics of $\mathcal{P}_{h^{N}}(\alpha, z)$ is given in Section 6 of [SoZ2].

Proof of Theorems 1-2:Let $S=\left\{\lambda^{1}, \ldots, \lambda^{f}\right\} \subset p \Sigma$. By Proposition 2.2 with $k=m$,

$$
\begin{aligned}
N^{-m} \mathbf{E}_{N \mid N S} Z_{p_{1}, \ldots, p_{m}} & =\left\{\frac{i}{2 \pi} \partial \bar{\partial}\left[\frac{1}{N} \log \Pi_{N p \mid N S}(z, z)\right]+\frac{p}{\pi} \omega_{\mathrm{FS}}\right\}^{m} \\
& =\operatorname{MA}\left\{\left[\frac{1}{N} \log \Pi_{N p \mid N S}(z, z)\right]+p \log \left(1+\|z\|^{2}\right)\right\} .
\end{aligned}
$$

By (38) and Lemma 2.6,

$$
\left[\frac{1}{N} \log \Pi_{N p \mid N S}(z, z)\right]+p \log \left(1+\|z\|^{2}\right) \rightarrow p \max _{\lambda \in S}\left[\left\langle\rho, \lambda^{p}\right\rangle-\left\langle\widehat{\lambda}^{p}, \log \widehat{\lambda}^{p}\right\rangle\right]+p \log p
$$

uniformly, where $\rho=\left(\log \left|z_{1}\right|^{2}, \ldots, \log \left|z_{m}\right|^{2}\right)$. Theorem 2 then follows from Proposition 2.2 and the Bedford-Taylor theorem [BT, Kl] on the continuity of the operator $\left(u_{1}, \ldots, u_{k}\right) \mapsto$ $d d^{c} u_{1} \wedge \cdots \wedge d d^{c} u_{k}$ under uniform limits. Theorem 1 is a special case of Theorem 2 .

Corollary3 follows immediately from Theorem 2 by averaging over the spectra in $\Delta$.

\section{Zeros of RAndom feWnomial systems: Proof of Theorem 4}

By Corollary 2.3 and the Bedford-Taylor continuity theorem for $d d^{c} u_{1} \wedge \cdots \wedge d d^{c} u_{k}$ under uniform limits, to prove Theorem 4 , it suffices to show that

$$
\begin{aligned}
\frac{1}{C(N, f)} \sum_{\sigma \in \mathcal{C}_{N, f}}\left(\frac{1}{N} \log \Pi_{N \mid \operatorname{Supp} \sigma}(z, z)\right. & \left.+\log \left(1+\|z\|^{2}\right)\right) \\
& \rightarrow \int_{\Sigma^{f}} \max _{j=1, \ldots, f}\left[\left\langle\rho, \lambda^{j}\right\rangle-\left\langle\widehat{\lambda^{j}}, \log \widehat{\lambda^{j}}\right\rangle\right] d \lambda^{1} \cdots d \lambda^{f}
\end{aligned}
$$

uniformly on compact subsets of $\left(\mathbb{C}^{*}\right)^{m}$.

We begin by writing the above sum as an integral. For $\alpha \in N \Sigma$, we write $\lfloor\alpha\rfloor=$ $\left(\left\lfloor\alpha_{1}\right\rfloor, \ldots,\left\lfloor\alpha_{m}\right\rfloor\right) \in N \Sigma \cap \mathbb{Z}^{m}$. For $\alpha=\left(\alpha^{1}, \ldots, \alpha^{f}\right) \in\left(\mathbb{Z}^{m} \cap N \Sigma\right)^{f}$, we consider the $m f$-cube of width $\frac{1}{N}$

Then

$$
R_{N, \alpha}:=\left\{\left(\lambda^{1}, \ldots, \lambda^{f}\right) \in\left(\mathbb{R}^{m}\right)^{f}:\left\lfloor N \lambda^{j}\right\rfloor=\alpha^{j}, 1 \leq j \leq f\right\}
$$

$$
\frac{(m !)^{f} f !}{N^{m f}} \sum_{\sigma \in \mathcal{C}_{N, f}} \log \Pi_{N \mid \operatorname{Supp} \sigma}(z, z)=\int_{U_{N}} \log \sum_{j=1}^{f}\left|\widehat{m}_{\left\lfloor N \lambda^{j}\right\rfloor}^{N}(z)\right|^{2} d \lambda^{1} \cdots d \lambda^{f},
$$


where $d \lambda^{j}=m ! d \lambda_{1}^{j} \cdots d \lambda_{m}^{j}$, and

$$
U_{N}=\bigcup\left\{R_{N, \alpha}: \alpha=\left(\alpha^{1}, \ldots, \alpha^{f}\right) \in\left(\mathbb{Z}^{m} \cap N \Sigma\right)^{f}, \alpha^{j} \neq \alpha^{j^{\prime}} \text { for } j \neq j^{\prime}\right\} .
$$

It then follows from (30) and the estimate $\operatorname{Vol}\left(\Sigma^{f} \triangle U_{N}\right)=O(1 / N)$ that

$$
\frac{1}{C(N, f)} \sum_{\sigma \in \mathcal{C}_{N, f}} \log \Pi_{N \mid \operatorname{Supp} \sigma}(z, z)=\int_{\Sigma^{f}} \log \sum_{j=1}^{f}\left|\widehat{m}_{\left\lfloor N \lambda^{j}\right\rfloor}^{N}(z)\right|^{2} d \lambda^{1} \cdots d \lambda^{f}+E_{N}(z),
$$

where

$$
\left|E_{N}(z)\right| \leq\left.\frac{C_{m}}{N} \max _{\beta^{j} \in \mathbb{Z}^{m} \cap N \Sigma}\left|\log \sum_{j=1}^{f}\right| \widehat{m}_{\beta^{j}}^{N}(z)\right|^{2} \mid .
$$

As in the proof of Lemma 2.6, we conclude from Lemma 2.5 that

$$
\max _{j}\left\{-N b_{\beta^{j} / N}(z)\right\}-C_{m}^{\prime} \leq \log \sum_{j=1}^{f}\left|\widehat{m}_{\beta^{j}}^{N}(z)\right|^{2} \leq \max _{j}\left\{-N b_{\beta^{j} / N}(z)\right\}+m \log N+C_{m}^{\prime}+\log f .
$$

Therefore, there are positive constants $C, C^{\prime}$ depending only on $m, f$ such that

$$
\left|E_{N}(z)\right| \leq C \sup _{\lambda \in \Sigma} b_{\lambda}(z)+C^{\prime}
$$

LEMMA 3.1. Let $\Psi: \Sigma^{f} \times\left(\mathbb{C}^{*}\right)^{m} \rightarrow \mathbb{R}$ be given by

$$
\Psi(\lambda, z)=\log \sum_{j=1}^{f}\left|\widehat{m}_{\left\lfloor N \lambda^{j}\right\rfloor}^{N}(z)\right|^{2}
$$

Then for all compact sets $K \subset\left(\mathbb{C}^{*}\right)^{m}$,

$$
\frac{1}{N} \Psi(\lambda, z) \rightarrow \max _{j}\left\{-b_{\lambda^{j}}(z)\right\} \quad \text { uniformly on } \Sigma^{f} \times K .
$$

Proof. Let $\varepsilon>0$ be arbitrary. By (44), we can choose $N_{0}$ such that

$$
\left.\left|\frac{1}{N} \log \sum_{j=1}^{f}\right| \widehat{m}_{\beta^{j}}^{N}(z)\right|^{2}-\max _{j}\left\{-b_{\beta^{j} / N}(z)\right\} \mid \leq \varepsilon \quad \forall \beta \in \Sigma^{f}, \forall z \in\left(\mathbb{C}^{*}\right)^{m}, \forall N \geq N_{0} .
$$

We can choose $N_{0}$ large enough so we also have

$$
|\alpha-\lambda|<\frac{1}{N_{0}} \Longrightarrow\left|b_{\alpha}(z)-b_{\lambda}(z)\right|<\varepsilon \quad \forall \alpha, \lambda \in \Sigma, \forall z \in K .
$$

Thus, for all $(\lambda, z) \in \Sigma^{f} \times K$ and $N>N_{0}$, we have

$$
\begin{aligned}
\left|\frac{1}{N} \Psi(\lambda, z)-\max _{j}\left\{-b_{\lambda^{j}}(z)\right\}\right| \leq \mid \frac{1}{N} \Psi(\lambda, z) & -\max _{j}\left\{-b_{\left\lfloor N \lambda^{j}\right\rfloor / N}(z) \mid\right. \\
& +\mid \max _{j}\left\{-b_{\left\lfloor N \lambda^{j}\right\rfloor / N}(z)-\max _{j}\left\{-b_{\lambda^{j}}(z)\right\} \mid<2 \varepsilon .\right.
\end{aligned}
$$


The desired uniform convergence (41) follows from (38), (43), (45), and Lemma 3.1, which completes the proof of Theorem 4 .

The same argument gives the proof of Theoem 6 .

3.1. Computing the explicit formula: Proof of Corollary 5. For $r \in \mathbb{R}^{m}$, we write $e^{r}=\left(e^{r_{1}}, \ldots, e^{r_{m}}\right)$, so that $\sum e^{\rho_{j}}=\left\|e^{\rho / 2}\right\|^{2}=\left|e^{\rho}\right|$.

Recalling (37), we write

$$
b(\lambda ; \rho):=b_{\{\lambda\}}\left(e^{\rho}\right)=\langle\widehat{\lambda}, \log \widehat{\lambda}\rangle-\langle\rho, \lambda\rangle+\log \left(1+\left|e^{\rho}\right|\right) \geq 0 .
$$

Therefore,

$$
\int_{\Sigma^{f}} \max _{j=1, \ldots, f}\left[\left\langle\rho, \lambda^{j}\right\rangle-\left\langle\widehat{\lambda}^{j}, \log \widehat{\lambda}^{j}\right\rangle\right] d \lambda^{1} \cdots d \lambda^{f}=\log \left(1+\left|e^{\rho}\right|\right)-\int_{\Sigma^{f}} \min _{j=1, \ldots, f} b\left(\lambda^{j} ; \rho\right) d \lambda^{1} \cdots d \lambda^{f} .
$$

We shall use the following elementary probability formula: Let $X$ be a non-negative random variable on a probability space $(\Omega, d P)$, and let $D_{X}(t):=P(X \leq t)$ be its distribution function. The expected value of $X$ is given by

$$
\mathbf{E}(X)=\int X d P=\int_{0}^{\infty} t d D_{X}(t)=\lim _{r \rightarrow \infty} \int_{0}^{r} t d D_{X}(t)
$$

where

$$
\int_{0}^{r} t d D_{X}(t)=r D_{X}(r)-\int_{0}^{r} D_{X}(t) d t=\int_{0}^{r}\left[D_{X}(r)-D_{X}(t)\right] d t .
$$

Letting $r \rightarrow \infty$, we have by Lebesgue monotone convergence

$$
\mathbf{E}(X)=\int X d P=\int_{0}^{\infty}\left[1-D_{X}(t)\right] d t
$$

We let

$$
D_{b}(t ; \rho):=P\{\lambda \in \Sigma: b(\lambda ; \rho) \leq t\}
$$

be the distribution function for $b(\cdot ; \rho)$, where $d P(\lambda)=m ! d \lambda_{1} \cdots d \lambda_{m}$. The distribution function for the random variable

$$
X\left(\lambda^{1}, \ldots, \lambda^{f}\right):=\min \left\{b\left(\lambda^{1}\right), \ldots, b\left(\lambda^{f}\right)\right\}
$$

on $\Sigma^{f}$ (with the product measure $\left.d P\left(\lambda^{1}\right) \cdots d P\left(\lambda^{f}\right)\right)$ is given by

$$
D_{X}=1-\left(1-D_{b}\right)^{f} .
$$

It then follows from (46)-(47) that

$$
\int_{\Sigma^{f}} \max _{j=1, \ldots, f}\left[\left\langle\rho, \lambda^{j}\right\rangle-\left\langle\widehat{\lambda}^{j}, \log \widehat{\lambda}^{j}\right\rangle\right] d \lambda^{1} \cdots d \lambda^{f}=\log \left(1+\left|e^{\rho}\right|\right)-\int_{0}^{\infty}\left[1-D_{b}(t ; \rho)\right]^{f} d t .
$$

Corollary 5 follows immediately from Theorem 4 and (48). 
3.1.1. The dimension 1 case. We now further evaluate $D_{b}$ when the dimension $m=1$. In this case,

$$
b(\lambda ; \rho)=\lambda \log \lambda+(1-\lambda) \log (1-\lambda)-\rho \lambda+\log \left(1+e^{\rho}\right), \quad 0 \leq \lambda \leq 1, \rho \in \mathbb{R} .
$$

Since $b$ is a convex function of $\lambda$ (taking the minimum value 0 when $\lambda=e^{\rho} /\left(1+e^{\rho}\right)$ ), we have $D_{b}(t ; \rho)=\widetilde{g}(t, \rho)-g(t, \rho)$ for $t \geq 0$, where $g(\cdot, \rho) \leq \widetilde{g}(\cdot, \rho)$ are the branches of $b(\cdot, \rho)^{-1}$. Precisely, $g=g(t, \rho), \widetilde{g}=\widetilde{g}(t, \rho)$ are given by

$$
\begin{array}{lll}
0 \leq g \leq \widetilde{g} \leq 1, & \\
b(g ; \rho)=t \quad \text { if } t \leq \log \left(1+e^{\rho}\right), & b(g ; \rho)=0 \quad \text { if } t>\log \left(1+e^{\rho}\right), \\
b(\widetilde{g} ; \rho)=t \quad \text { if } t \leq \log \left(1+e^{-\rho}\right), & b(\widetilde{g} ; \rho)=1 \quad \text { if } t>\log \left(1+e^{-\rho}\right) .
\end{array}
$$

We have the symmetry $b(\lambda ; \rho)=b(1-\lambda ;-\rho)$, and hence $\widetilde{g}(t, \rho)=1-g(t,-\rho)$. Therefore,

$$
D_{b}(t ; \rho)=1-g(t, \rho)-g(t,-\rho),
$$

where $g(\cdot, \rho):[0,+\infty) \rightarrow\left[0, e^{\rho} /\left(1+e^{\rho}\right)\right]$ is given by:

$$
\begin{array}{ll}
b(g(t, \rho), \rho)=t, & \text { if } 0 \leq t \leq \log \left(1+e^{\rho}\right), \\
g(t, \rho)=0, & \text { if } t \geq \log \left(1+e^{\rho}\right) .
\end{array}
$$

\section{General toric KÄHLER Potentials}

We now sketch the proof of Theorem 7. It is almost the same as in the Fubini-Study case but requires the generalization of Lemma 2.5 and then Lemma 2.6.

As discussed in [SoZ2], the toric norming constants can be written in terms of the symplectic potential as follows:

$$
Q_{G_{N}\left(\varphi, d V_{\varphi}\right)}(\alpha)=\int_{P} e^{-N\left(u_{\varphi}(x)+\left\langle\frac{\alpha}{N}-x, \log \mu_{\varphi}^{-1}(x)\right\rangle\right.} d x .
$$

Here, $\mu_{\varphi}\left(e^{\rho / 2}\right)=\nabla_{\rho} \varphi\left(e^{\rho / 2}\right)$ is the moment map determined by $\varphi$. Applying steepest descent to the integral, we find that there exists only one critical point at $x=\mu_{\varphi}\left(e^{\rho / 2}\right)$, and we conclude that

$$
\frac{1}{N} \log Q_{G_{N}\left(\varphi, d V_{\varphi}\right)}(\alpha)=u_{\varphi}\left(\frac{\alpha}{N}\right)+O\left(\frac{\log N}{N}\right)
$$

uniformly [SoZ3, (25)].

The logarithmic asymptotics (52) is the only non-obvious aspect of the logarithmic mass asymptotics. The Szegö kernel for a single lattice point (on the diagonal) equals

$$
\Pi_{N, Q \mid \alpha}\left(e^{\rho / 2}, e^{\rho / 2}\right)=\frac{e^{\langle\alpha, \rho\rangle} e^{-N \varphi\left(e^{\rho / 2}\right)}}{Q_{G_{N}\left(\varphi, d V_{\varphi}\right)}} .
$$

The analogue of Lemma 2.5 for a general Kähler potential is

$$
\log \Pi_{N, Q \mid N x}\left(e^{\rho / 2}, e^{\rho / 2}\right)=N\left(\langle x, \rho\rangle-\varphi\left(e^{\rho / 2}\right)-u_{\varphi}(x)\right)+O(\log N),
$$

which follows from (52) and [SoZ3, (55)].

For a fewnomial Szegö kernel with a finite set $S$ of lattice points, the analogue of Lemma 2.6 is that

$$
\frac{1}{N} \log \Pi_{N, Q \mid N S}\left(e^{\rho / 2}, e^{\rho / 2}\right)=\max _{\lambda \in S}\left(\langle\lambda, \rho\rangle-u_{\varphi}(\lambda)\right)-\varphi\left(e^{\rho / 2}\right)+O\left(\frac{\log N}{N}\right) .
$$


The proof is the same as that of Lemma 2.6, using (53). With this modification, the remainder of the proof of Theorem 7 is the same as that of Theorem 4 .

\section{The $\mathbf{T}^{m}$ ENSEMBLE}

Finally, we indicate the modifications needed to deal with the fewnomial Kac-Hammersley ensemble (10) . This is quite different from the case of pluri-subharmonic weights because the Szegö kernel has quite different (much weaker) asymptotic properties. But for fewnomial Szegö kernels the distinction is not too severe.

In this case, we use the $L^{2}$ norm $\|\cdot\|_{\mathbf{T}^{m}}$ on the real torus rather than the Fubini-Study norm. We therefore have

$$
\mathbf{E}_{\mathbf{T}^{m} \mid S}\left(|P(z)|_{\mathbf{T}^{m}}^{2}\right)=\sum_{\alpha, \beta \in S} \mathbf{E}\left(\lambda_{\alpha} \bar{\lambda}_{\beta}\right) \chi_{\alpha}(z) \overline{\chi_{\beta}(z)}
$$

Since $\mathbf{E}\left(\lambda_{\alpha} \bar{\lambda}_{\beta}\right)=\delta_{\alpha}^{\beta}$, we have:

$$
\mathbf{E}_{\mathbf{T}^{m} \mid S}\left(|P(z)|_{\mathbf{T}^{m}}^{2}\right)=\sum_{\alpha \in S}\left|\chi_{\alpha}(z)\right|^{2}=\Pi_{\mathbf{T}^{m} \mid S}(z, z)
$$

where $\Pi_{\mathbf{T}^{m} \mid S}$ is the orthogonal projection onto $\operatorname{Poly}(S) \subset L^{2}\left(\left(\mathbb{C}^{*}\right)^{m}, \delta_{\mathbf{T}^{m}}\right)$. It then follows by expressing the Gaussian in spherical coordinates that the expectation in the fewnomial Kac-Hammersley ensemble is given by

$$
\mathbf{E}_{K H}\left(|P(z)|_{\mathbf{T}^{m}}^{2}\right)=\frac{1}{\# S} \mathbf{E}_{K H}\left(|P(z)|_{\mathbf{T}^{m}}^{2}\right)=\frac{1}{\# S} \Pi_{\mathbf{T}^{m} \mid S}(z, z) .
$$

It is clear that

$$
\Pi_{\mathbf{T}^{m} \mid S}(z, w)=\sum_{\alpha \in S}\langle z, \bar{w}\rangle^{\alpha}
$$

Therefore,

$$
\Pi_{\mathbf{T}^{m}, S}(z, z)=\sum_{\alpha \in S}\left|z^{\alpha}\right|^{2}=\sum_{\alpha \in S} e^{\langle\rho, \alpha\rangle}, \quad z=e^{i \varphi+\rho / 2} .
$$

The potential in this case is

$$
F_{N}^{f}(z):=\frac{1}{C(N, f)} \sum_{S \in \mathcal{F}_{N, f}} \log \Pi_{\mathbf{T}^{m}, S}(z, z)
$$

Proposition 5.1.

$$
\lim _{N \rightarrow \infty} \frac{1}{N} F_{N}^{f}\left(e^{\rho / 2}\right)=\int_{\Sigma^{f}} \max \left\{\left\langle x_{1}, \rho\right\rangle \ldots,\left\langle x_{f}, \rho\right\rangle\right\} d x_{1} \cdots d x_{f} .
$$

Outline of the proof: Indeed,

$$
\log \sum_{\alpha \in S} e^{\langle\rho, \alpha\rangle}=N \log \sum_{\alpha \in S} e^{\langle\rho, \alpha / N\rangle} \sim N \max _{\alpha \in S}\{\langle\rho, \alpha / N\rangle\} .
$$

Hence,

$$
F_{N}^{f}\left(e^{\rho}\right)=\frac{1}{C(N, f)} \sum_{S \in \mathcal{F}_{N, f}} \log \sum_{\alpha \in S} e^{\langle\rho, \alpha\rangle} \sim N \int_{\Sigma^{f}} \max \left\{\left\langle x^{1}, \rho\right\rangle \ldots,\left\langle x^{f}, \rho\right\rangle\right\} d x^{1} \cdots d x^{f} .
$$


We note that for each $\left(x^{1}, \ldots, x^{f}\right)$, the function $M_{\left(x^{1}, \ldots, x^{m}\right)}(\rho)=\max \left\{\left\langle x^{1}, \rho\right\rangle \ldots,\left\langle x^{f}, \rho\right\rangle\right\}$ is a piecewise linear convex function. It follows that the integral defines a convex function of $\rho$.

In dimension one, if all $x^{j} \geq 0$,

$$
\max \left\{\rho x^{1}, \ldots, \rho x^{f}\right)=\left\{\begin{array}{cl}
\rho \max \left\{x^{1}, \ldots, x^{f}\right\}, & \rho \geq 0 \\
\rho \min \left\{x^{1}, \ldots, x^{f}\right\}, & \rho \leq 0
\end{array}\right.
$$

Hence,

$$
F_{N}^{k}\left(e^{\rho}\right) \sim \begin{cases}N \rho\left\{\int_{[0,1]^{f}} \max \left\{x^{1}, \ldots, x^{f}\right\} d x^{1} \cdots d x^{f}\right\}, & \rho \geq 0 \\ \left.N \rho\left\{\int_{[0,1]^{f}} \min \left\{x^{1}, \ldots, x^{f}\right\}\right\} d x^{1} \cdots d x^{f}\right\}, & \rho \leq 0\end{cases}
$$

Thus, $F_{N}^{k}\left(e^{\rho}\right)$ is piecewise linear in $\rho$ with a corner at $\rho=0$. In dimension one,

$$
\frac{1}{N} \mathbf{E}_{N, f}\left(Z_{P^{N}}\right)=\frac{\sqrt{-1}}{2 \pi N} \partial \bar{\partial} F_{N}^{k} \rightarrow \delta_{S^{1}}
$$

\section{REFERENCES}

[BBS] D. J. Bates, F. Bihan and F. Sottile, Bounds on the number of real solutions to polynomial equations. Int. Math. Res. Not. IMRN 2007, no. 23, Art. ID rnm114, 7 pp.

[BT] E. Bedford and B. A. Taylor, A new capacity for plurisubharmonic functions, Acta Math. 149 (1982), $1-40$.

[Be] D. N. Bernstein, The number of roots of a system of equations, Functional Anal. Appl. 9 (1975), $183-185$.

[BRS] F. Bihan, J. M. Rojas and F. Sottile, On the sharpness of fewnomial bounds and the number of components of fewnomial hypersurfaces. Algorithms in algebraic geometry, 1520, IMA Vol. Math. Appl., 146, Springer, New York, 2008.

[BS] T. Bloom and B. Shiffman, Zeros of random polynomials on $\mathbb{C}^{m}$, Math. Res. Lett. 14 (2007), 469-479.

$[\mathrm{Bu}] \quad$ P. Bürgisser, Average Euler characteristic of random real algebraic varieties. C. R. Math. Acad. Sci. Paris 345 (2007), no. 9, 507-512.

[GW] D. Gayet and J.-Y. Welschinger, Exponential rarefaction of real curves with many components, (arXiv:1005.3228v1).

[Ha] J. M. Hammersley, The zeros of a random polynomial, Proceedings of the Third Berkeley Symposium on Mathematical Statistics and Probability, 1954-1955, vol. II, 89-111, University of California Press, Berkeley and Los Angeles, 1956.

[Ka1] B. Ya. Kazarnovskii, On zeros of exponential sums. (Russian) Dokl. Akad. Nauk SSSR 257 (1981), no. $4,804-808$.

[Ka2] B. Ya. Kazarnovskii, Newton polyhedra and roots of systems of exponential sums. (Russian) Funktsional. Anal. i Prilozhen. 18 (1984), no. 4, 40-49, 96.

[Kh] A. G. Khovanskii, Fewnomials, Trans. Math Monographs 88, AMS Publications, Providence, Rhode Island, 1991.

[Kl] M. Klimek, Pluripotential Theory, London Math. Soc. Monographs, New Series 6, Oxford University Press, New York, 1991.

[Ko] A. G. Kouchnirenko, Polyèdres de Newton et nombres de Milnor, Invent. Math. 32 (1976), 1-31.

[Ro] J. M. Rojas, On the average number of real roots of certain random sparse polynomial systems. In: The mathematics of numerical analysis (Park City, UT, 1995), 689-699, Lectures in Appl. Math. 32, Amer. Math. Soc., Providence, RI, 1996.

[STZ] B. Shiffman, T. Tate and S. Zelditch, Distribution laws for integrable eigenfunctions. Ann. Inst. Fourier 54 (2004), no. 5, 1497-1546. 
[SZ1] B. Shiffman and S. Zelditch, Distribution of zeros of random and quantum chaotic sections of positive line bundles, Comm. Math. Phys. 200 (1999), 661-683.

[SZ2] B. Shiffman and S. Zelditch, Random polynomials with prescribed Newton polytope, J. Amer. Math. Soc. 17 (2004), no. 1, 49-108.

[SZ3] B. Shiffman and S. Zelditch, Number variance of random zeros on complex manifolds, Geom. Funct. Anal. 18, No. 4 (2008), 1422-1475.

[SZZ] B. Shiffman, S. Zelditch and Q. Zhong, Random zeros on complex manifolds: conditional expectations, (arXiv:1005.4166v1).

[ShSm] M. Shub and S. Smale, Complexity of Bezout's theorem. II. Volumes and probabilities. Computational algebraic geometry (Nice, 1992), 267-285, Progr. Math., 109, Birkhäuser Boston, Boston, MA, 1993.

[SoZ1] J. Song and S. Zelditch, Convergence of Bergman geodesics on $\mathbf{C P}^{1}$, Ann. Inst. Fourier 57, no. 6 (Festival Colin de Verdière) (2007), 2209-2237.

[SoZ2] J. Song and S. Zelditch, Bergman metrics and geodesics in the space of Kähler metrics on toric varieties, Analysis \& PDE 3, No. 2 (2010), 295-358.

[SoZ3] J. Song and S. Zelditch, Test configurations, large deviations and geodesic rays on toric varieties, (arXiv:0712.3599).

[Sot] F. Sottile, Enumerative real algebraic geometry. Algorithmic and quantitative real algebraic geometry (Piscataway, NJ, 2001), 139-179, DIMACS Ser. Discrete Math. Theoret. Comput. Sci., 60, Amer. Math. Soc., Providence, RI, 2003.

[St1] B. Sturmfels, On the number of real roots of a sparse polynomial system, Hamiltonian and gradient flows, algorithms and control, Fields Inst. Commun. 3, Amer. Math. Soc., Providence, RI, 1994, pp. 137-143.

[St2] B. Sturmfels, Polynomial equations and convex polytopes. Amer. Math. Monthly 105 (1998), no. 10, 907-922.

Department of Mathematics, Johns Hopkins University, Baltimore, MD 21218, USA

E-mail address: shiffman@math.jhu.edu

Department of Mathematics, Northwestern University, Evanston, IL 60208, USA

E-mail address: zelditch@math.northwestern.edu 